\title{
SOME FIXED POINT THEOREMS VIA COMMON LIMIT RANGE PROPERTY IN NON-ARCHIMEDEAN MENGER PROBABILISTIC METRIC SPACES
}

\author{
Hemant Kumar Nashine and Zoran Kadelburg
}

\begin{abstract}
We propose coincidence and common fixed point results for a quadruple of self mappings satisfying common limit range property and weakly compatibility under generalized $\Phi$-contractive conditions in Non-Archimedean Menger PM-spaces. As examples we exhibit different types of situations where these conditions can be used. A common fixed point theorem for four finite families of self mappings is presented as an application of the proposed results. The existence and uniqueness of solutions for certain system of functional equations arising in dynamic programming are also presented as another application.
\end{abstract}

\section{Introduction}

The notion of probabilistic metric space (briefly, PM-space) as a generalization of metric space, was introduced in 1942 by K. Menger. The first idea of Menger was to use distribution functions instead of non-negative real numbers as values of the metric. Such a probabilistic generalization of metric spaces appears to be well adapted for the investigation of physical quantities and physiological thresholds. It is also of fundamental importance in probabilistic functional analysis. Since then the theory of probabilistic metric spaces has been developed in many directions.

Non-Archimedean probabilistic metric spaces (briefly, N. A. PM-spaces) and some of their topological properties were first studied by Istrătescu and Crivăt [21] in the year 1974. Istrătescu $[18,19]$ obtained some fixed point theorems on N. A. Menger PM-spaces and generalized the results of Sehgal and BharuchaReid [31] (see also [20, 22]). Further, Hadžić [13] improved the results of Istrătescu $[18,19]$. The theory of probabilistic metric spaces is of fundamental importance in probabilistic functional analysis due to its extensive applications in random differential as well as random integral equations (see $[5,12])$.

Received January 19, 2014.

2010 Mathematics Subject Classification. 47H10, 54H25.

Key words and phrases. t-norm, non-Archimedean Menger PM-space, weakly compatible mappings, common limit range property, fixed point. 
In 1987, Singh and Pant [34] introduced the notion of weakly commuting mappings on N. A. Menger PM-spaces and proved some common fixed point theorems. Dimri and Pant [11] studied the application of N. A. Menger PMspaces to product spaces. Jungck and Rhoades [23, 24] weakened the notion of compatible mappings by introducing weakly compatible mappings and proved common fixed point theorems without any requirement of continuity of the involved mappings. Many mathematicians proved common fixed point theorems in N. A. Menger PM-spaces using different contractive conditions (see $[4,10,11,25,26,27,32,33,36])$.

In 2002, Aamri and Moutawakil [1] defined the notion of property (E.A) which contained the class of non-compatible mappings. It is observed that the property (E.A) requires the completeness (or closedness) of the subspaces for the existence of a common fixed point. As a further generalization, new notion of CLRg property, recently given by Sintunavarat and Kuman [37], does not impose such conditions. The importance of CLRg property is that it ensures that one does not require the closedness of range of subspaces (see also [38]). This concept was used by Singh et al. [35] who proved a common fixed point theorem for a pair of weakly compatible self mappings in an N. A. Menger PM-space employing common limit range property. Recently, Imdad et al. [17] extended the notion of common limit range property to two pairs of self mappings which further relaxes the requirement on closedness of the subspaces. Since then, a number of fixed point theorems has been established by several researchers in different settings under common limit range property. We refer the reader to $[15,39]$ and references therein. Further, using this concept for two pairs in N. A. Menger PM-spaces, Chauhan and Vujaković [8] extended results of Singh et al. [35].

The proposed results will be explained in the further sections, but we state here briefly some improvements that we intend to achieve: (i) Containment of ranges amongst the involved mappings is relaxed. (ii) Continuity requirements of all the involved mappings are completely relaxed. (iii) The (E.A) property is replaced by $\left(C L R_{S, T}\right)$ property which is the most general among all existing weak commutativity concepts. (iv) The condition on completeness of the whole space is relaxed.

In the final two sections, we present examples that exhibit different types of situations where the obtained results can be used; moreover, the existence and uniqueness of solutions for a certain system of functional equations arising in dynamic programming are also presented as another application.

\section{Preliminaries}

We shall recall some definitions and mathematical preliminaries.

Definition ([30]). A triangular norm (briefly a $t$-norm) $\mathcal{T}$ is a binary operation on the unit interval $[0,1]$ such that for all $a, b, c, d \in[0,1]$ the following conditions are satisfied: 
(1) $\mathcal{T}(a, 1)=a$ for all $a \in[0,1]$;

(2) $\mathcal{T}(a, b)=\mathcal{T}(b, a)$

(3) $\mathcal{T}(a, b) \leq \mathcal{T}(c, d)$, whenever $a \leq c$ and $b \leq d$;

(4) $\mathcal{T}(a, \mathcal{T}(b, c))=\mathcal{T}(\mathcal{T}(a, b), c)$

Some examples of $t$-norms are $\mathcal{T}(a, b)=\min \{a, b\}, \mathcal{T}(a, b)=a b$ and $\mathcal{T}(a, b)=$ $\max \{a+b-1,0\}$.

Definition ([30]). A mapping $F: \mathbb{R} \rightarrow \mathbb{R}^{+}$is said to be a distribution function if it is non-decreasing and left continuous with $\inf \{F(t): t \in \mathbb{R}\}=0$ and $\sup \{F(t): t \in \mathbb{R}\}=1$.

We shall denote by $\Im$ the set of all distribution functions while $H$ will always denote the specific distribution function defined by

$$
H(t)= \begin{cases}0, & \text { if } t \leq 0, \\ 1, & \text { if } t>0 .\end{cases}
$$

If $X$ is a non-empty set, $\mathcal{F}: X \times X \rightarrow \Im$ is called a probabilistic distance on $X$ and $\mathcal{F}(x, y)$ is usually denoted by $F_{x, y}$.

Definition $([19,21])$. The ordered pair $(X, \mathcal{F})$ is said to be an N. A. PM-space if $X$ is a non-empty set and $\mathcal{F}$ is a probabilistic distance satisfying the following conditions: for all $x, y, z \in X$ and $t, t_{1}, t_{2}>0$,

(1) $F_{x, y}(t)=1 \Longleftrightarrow x=y$;

(2) $F_{x, y}(t)=F_{y, x}(t)$;

(3) if $F_{x, y}\left(t_{1}\right)=1$ and $F_{y, z}\left(t_{2}\right)=1$, then $F_{x, z}\left(\max \left\{t_{1}, t_{2}\right\}\right)=1$.

The ordered triplet $(X, \mathcal{F}, \mathcal{T})$ is called an N. A. Menger PM-space if $(X, \mathcal{F})$ is an N. A. PM-space, $\mathcal{T}$ is a $t$-norm and the following inequality holds:

$$
F_{x, z}\left(\max \left\{t_{1}, t_{2}\right\}\right) \geq \mathcal{T}\left(F_{x, y}\left(t_{1}\right), F_{y, z}\left(t_{2}\right)\right)
$$

for all $x, y, z \in X$ and $t_{1}, t_{2}>0$.

The concept of neighbourhoods in Menger PM-spaces was introduced by Schweizer and Sklar [30]. If $x \in X, \epsilon>0$ and $\lambda \in(0,1)$, then an $(\epsilon, \lambda)$ neighbourhood of $x, U_{x}(\epsilon, \lambda)$ is defined by

$$
U_{x}(\epsilon, \lambda)=\left\{y \in X: F_{x, y}(\epsilon)>1-\lambda\right\} .
$$

If the $t$-norm $\mathcal{T}$ is continuous and strictly increasing, then $(X, \mathcal{F}, \mathcal{T})$ is a Hausdorff space in the topology induced by the family $\left\{U_{x}(\epsilon, \lambda): x \in X, \epsilon>0, \lambda \in\right.$ $(0,1)\}$ of neighbourhoods [30].

Example 2.1. Let $X$ be any set with at least two elements. If we define $F_{x, x}(t)=1$ for all $x \in X, t>0$ and

$$
F_{x, y}(t)= \begin{cases}0, & \text { if } t \leq 1 \\ 1, & \text { if } t>1\end{cases}
$$

where $x, y \in X, x \neq y$, then $(X, \mathcal{F}, \mathcal{T})$ is an N. A. Menger PM-space with $\mathcal{T}(a, b)=\min \{a, b\}$ or $(a b)$ for all $a, b \in[0,1]$. 
Example 2.2. Let $X=\mathbb{R}$ be the set of real numbers equipped with the metric defined by $d(x, y)=|x-y|$ and

$$
F_{x, y}(t)= \begin{cases}\frac{t}{t+|x-y|}, & \text { if } t>0 \\ 0, & \text { if } t=0 .\end{cases}
$$

Then $(X, \mathcal{F}, \mathcal{T})$ is an N. A. Menger PM-space with $\mathcal{T}$ as continuous $t$-norm satisfying $\mathcal{T}(a, b)=\min \{a, b\}$ or $(a b)$ for all $a, b \in[0,1]$.

Let us denote $\Omega=\{\mathfrak{g} \mid \mathfrak{g}:[0,1] \rightarrow[0, \infty)$ is continuous, strictly decreasing with $\mathfrak{g}(1)=0$ and $\mathfrak{g}(0)<\infty\}$.

Definition $([10])$. Let $\mathfrak{g} \in \Omega$. An N. A. Menger PM-space $(X, \mathcal{F}, \mathcal{T})$ is said to be of type $(C)_{\mathfrak{g}}$ if

$$
\mathfrak{g}\left(F_{x, z}(t)\right) \leq \mathfrak{g}\left(F_{x, y}(t)\right)+\mathfrak{g}\left(F_{y, z}(t)\right)
$$

for all $x, y, z \in X, t \geq 0$,

Definition $([10])$. Let $\mathfrak{g} \in \Omega$. An N. A. Menger PM-space $(X, \mathcal{F}, \mathcal{T})$ is said to be of type $(D)_{\mathfrak{g}}$ if

for all $t_{1}, t_{2} \in[0,1]$.

$$
\mathfrak{g}\left(\mathcal{T}\left(t_{1}, t_{2}\right)\right) \leq \mathfrak{g}\left(t_{1}\right)+\mathfrak{g}\left(t_{2}\right)
$$

Remark $2.3([10])$. If an N. A. Menger PM-space $(X, \mathcal{F}, \mathcal{T})$ is of type $(D)_{\mathfrak{g}}$, then

(1) it is of type $(C)_{\mathfrak{g}}$;

(2) it is metrizable, where the metric $d$ on $X$ is defined by

$$
d(x, y)=\int_{0}^{1} \mathfrak{g}\left(F_{x, y}(t)\right) d t
$$

for all $x, y \in X$.

Throughout this paper $(X, \mathcal{F}, \mathcal{T})$ will be an N. A. Menger PM-space with a continuous strictly increasing $t$-norm $\mathcal{T}$.

Definition ([9]). Two self mappings $A$ and $S$ of an N. A. Menger PM-space $(X, \mathcal{F}, \mathcal{T})$ are said to be compatible if $\lim _{n \rightarrow \infty} \mathfrak{g}\left(F_{A S x_{n}, S A x_{n}}(t)\right)=0$ for all $t>0$ and $\mathfrak{g} \in \Omega$, whenever $\left\{x_{n}\right\}$ is a sequence in $X$ such that $\lim _{n \rightarrow \infty} A x_{n}=$ $\lim _{n \rightarrow \infty} S x_{n}=z$ for some $z \in X$.

Definition. A pair $(A, S)$ of self mappings of an N. A. Menger PM-space $(X, \mathcal{F}, \mathcal{T})$ is said to satisfy (E.A) property if there exists a sequence $\left\{x_{n}\right\}$ in $X$ such that

for some $z \in X$.

$$
\lim _{n \rightarrow \infty} A x_{n}=\lim _{n \rightarrow \infty} S x_{n}=z
$$

Definition ([23]). A pair $(A, S)$ of self mappings of a non-empty set $X$ is said to be weakly compatible (or coincidentally commuting) if they commute at their coincidence points, i.e., if $A z=S z$ for some $z \in X$, then $A S z=S A z$. 
If two self mappings $A$ and $S$ of an N. A. Menger PM-space $(X, \mathcal{F}, \mathcal{T})$ are compatible then they are weakly compatible but the converse need not be true (see [29, Example 12]). It can be noticed that the notions of weak compatibility and property (E.A) are independent to each other [28, Example 2.2].

Definition. Two pairs $(A, S)$ and $(B, T)$ of self mappings of an N. A. Menger PM-space $(X, \mathcal{F}, \mathcal{T})$ are said to satisfy the common property $(E . A)$, if there exist two sequences $\left\{x_{n}\right\},\left\{y_{n}\right\}$ in $X$ for some $z$ in $X$ such that

$$
\lim _{n \rightarrow \infty} A x_{n}=\lim _{n \rightarrow \infty} S x_{n}=\lim _{n \rightarrow \infty} B y_{n}=\lim _{n \rightarrow \infty} T y_{n}=z \text {. }
$$

Definition ([37]). A pair $(A, S)$ of self mappings of an N. A. Menger PM-space $(X, \mathcal{F}, \mathcal{T})$ is said to satisfy the common limit range property with respect to mapping $S$, denoted by $\left(C L R_{S}\right)$, if there exists a sequence $\left\{x_{n}\right\}$ in $X$ such that

$$
\lim _{n \rightarrow \infty} A x_{n}=\lim _{n \rightarrow \infty} S x_{n}=z
$$

for some $z \in S(X)$.

Definition ([8]). Two pairs $(A, S)$ and $(B, T)$ of self mappings of an N. A. Menger PM-space $(X, \mathcal{F}, \mathcal{T})$ are said to satisfy the common limit range property with respect to mappings $S$ and $T$, denoted by $\left(C L R_{S T}\right)$, if there exist two sequences $\left\{x_{n}\right\}$ and $\left\{y_{n}\right\}$ in $X$ such that

$$
\lim _{n \rightarrow \infty} A x_{n}=\lim _{n \rightarrow \infty} S x_{n}=\lim _{n \rightarrow \infty} B y_{n}=\lim _{n \rightarrow \infty} T y_{n}=z,
$$

where $z \in S(X) \cap T(X)$.

Definition ([17]). Two families of self mappings $\left\{A_{i}\right\}$ and $\left\{S_{j}\right\}$ are said to be pairwise commuting if:

(1) $A_{i} A_{j}=A_{j} A_{i}, i, j \in\{1,2, \ldots, m\}$,

(2) $S_{k} S_{l}=S_{l} S_{k}, k, l \in\{1,2, \ldots, n\}$,

(3) $A_{i} S_{k}=S_{k} A_{i}, i \in\{1,2, \ldots, m\}, k \in\{1,2, \ldots, n\}$.

\section{Main results}

In what follows, we denote by $\Phi$ the collection of all functions $\varphi:[0, \infty) \rightarrow$ $[0, \infty)$ which are upper semicontinuous from the right and satisfy $\varphi(t)<t$, for all $t>0$.

For completion of our results, we need the following lemma.

Lemma $3.1([10])$. If a function $\phi:[0, \infty) \rightarrow[0, \infty)$ belongs to the class $\Phi$, then we have:

(1) for all $t \geq 0, \lim _{n \rightarrow \infty} \phi^{n}(t)=0$, where $\phi^{n}(t)$ is the $n^{\text {th }}$ iteration of $\phi(t)$

(2) if $\left\{t_{n}\right\}$ is a non-decreasing sequence of real numbers and $t_{n+1} \leq \phi\left(t_{n}\right)$ where $n=1,2, \ldots$, then $\lim _{n \rightarrow \infty} t_{n}=0$. In particular, if $t \leq \phi(t)$ for each $t \geq 0$, then $t=0$.

Now we state and prove our first main result. 
Theorem 3.2. Let $A, B, S$ and $T$ be four self mappings of an $N$. A. Menger PM-space $(X, \mathcal{F}, \mathcal{T})$, where $\mathcal{T}$ is a continuous t-norm, satisfying

$$
\begin{aligned}
& \mathfrak{g}\left(F_{A x, B y}(t)\right) \\
\leq & \phi\left(\max \left\{\begin{array}{c}
\mathfrak{g}\left(F_{S x, T y}(t)\right), \mathfrak{g}\left(F_{S x, A x}(t)\right), \mathfrak{g}\left(F_{T y, B y}(t)\right), \\
\frac{1}{2}\left[\mathfrak{g}\left(F_{A x, S x}(t)\right)+\mathfrak{g}\left(F_{B y, T y}(t)\right)\right], \frac{1}{2}\left[\mathfrak{g}\left(F_{A x, S x}(t)\right)+\mathfrak{g}\left(F_{S x, T y}(t)\right)\right], \\
\frac{1}{2}\left[\mathfrak{g}\left(F_{B y, T y}(t)\right)+\mathfrak{g}\left(F_{S x, T y}(t)\right)\right], \frac{1}{2}\left[\mathfrak{g}\left(F_{S x, B y}(t)\right)+\mathfrak{g}\left(F_{T y, A x}(t)\right)\right]
\end{array}\right\}\right)
\end{aligned}
$$

for all $x, y \in X, t>0$, where $\mathfrak{g} \in \Omega$ and $\phi \in \Phi$.

If the pairs $(A, S)$ and $(B, T)$ share the $\left(C L R_{S T}\right)$ property, then $(A, S)$ and $(B, T)$ have a coincidence point each. Moreover, $A, B, S$ and $T$ have a unique common fixed point provided both pairs $(A, S)$ and $(B, T)$ are weakly compatible.

Proof. In view of the fact that the pairs $(A, S)$ and $(B, T)$ share the $\left(C L R_{S T}\right)$ property, there exist two sequences $\left\{x_{n}\right\}$ and $\left\{y_{n}\right\}$ in $X$ such that

$$
\lim _{n \rightarrow \infty} A x_{n}=\lim _{n \rightarrow \infty} S x_{n}=\lim _{n \rightarrow \infty} T y_{n}=\lim _{n \rightarrow \infty} B y_{n}=z,
$$

where $z \in S(X) \cap T(X)$. As $z \in S(X)$, there exists a point $v \in X$ such that $S v=z$. First we assert that $A v=S v$. On using inequality (3.1) with $x=v$, $y=y_{n}$, we get

$$
\begin{aligned}
& \mathfrak{g}\left(F_{A v, B y_{n}}(t)\right) \\
\leq & \phi\left(\max \left\{\begin{array}{c}
\mathfrak{g}\left(F_{S v, T y_{n}}(t)\right), \mathfrak{g}\left(F_{S v, A v}(t)\right), \mathfrak{g}\left(F_{T y_{n}, B y_{n}}(t)\right), \\
\frac{1}{2}\left[\mathfrak{g}\left(F_{A v, S v}(t)\right)+\mathfrak{g}\left(F_{B y_{n}, T y_{n}}(t)\right)\right], \frac{1}{2}\left[\mathfrak{g}\left(F_{A v, S v}(t)\right)+\mathfrak{g}\left(F_{S v, T y_{n}}(t)\right)\right], \\
\frac{1}{2}\left[\mathfrak{g}\left(F_{B y_{n}, T y_{n}}(t)\right)+\mathfrak{g}\left(F_{S v, T y_{n}}(t)\right)\right], \frac{1}{2}\left(\mathfrak{g}\left(F_{S v, B y_{n}}(t)\right)+\mathfrak{g}\left(F_{T y_{n}, A v}(t)\right)\right)
\end{array}\right\}\right) .
\end{aligned}
$$

Passing to the limit as $n \rightarrow \infty$, this reduces to

$$
\begin{aligned}
& \mathfrak{g}\left(F_{A v, z}(t)\right) \\
\leq & \phi\left(\max \left\{\begin{array}{c}
\mathfrak{g}\left(F_{z, z}(t)\right), \mathfrak{g}\left(F_{z, A v}(t)\right), \mathfrak{g}\left(F_{z, z}(t)\right), \\
\frac{1}{2}\left[\mathfrak{g}\left(F_{A v, S v}(t)\right)+\mathfrak{g}\left(F_{z, z}(t)\right)\right], \frac{1}{2}\left[\mathfrak{g}\left(F_{A v, S v}(t)\right)+\mathfrak{g}\left(F_{S v, z}(t)\right)\right], \\
\frac{1}{2}\left[\mathfrak{g}\left(F_{z, z}(t)\right)+\mathfrak{g}\left(F_{S v, z}(t)\right)\right], \frac{1}{2}\left(\mathfrak{g}\left(F_{z, z}(t)\right)+\mathfrak{g}\left(F_{z, A v}(t)\right)\right)
\end{array}\right\}\right) \\
= & \phi\left(\max \left\{\begin{array}{c}
\mathfrak{g}(1), \mathfrak{g}\left(F_{z, A v}(t)\right), \mathfrak{g}(1), \frac{1}{2}[\mathfrak{g}(1)+\mathfrak{g}(1)], \frac{1}{2}\left[\mathfrak{g}(1)+\mathfrak{g}\left(F_{A v, z}(t)\right)\right], \\
\frac{1}{2}\left[\mathfrak{g}(1)+\mathfrak{g}\left(F_{A v, z}(t)\right)\right], \frac{1}{2}\left(\mathfrak{g}(1)+\mathfrak{g}\left(F_{z, A v}(t)\right)\right)
\end{array}\right\}\right) \\
= & \phi\left(\max \left\{\begin{array}{c}
\left.\left.0, \mathfrak{g}\left(F_{z, A v}(t)\right), 0,0, \frac{1}{2} \mathfrak{g}\left(F_{A v, z}(t)\right), \frac{1}{2} \mathfrak{g}\left(F_{A v, z}(t)\right), \frac{1}{2} \mathfrak{g}\left(F_{z, A v}(t)\right)\right\}\right) \\
=
\end{array}\right)\right. \\
& \phi\left(\mathfrak{g}\left(F_{z, A v}(t)\right)\right) .
\end{aligned}
$$

Making use of Lemma 3.1, we get $A v=S v=z$, which shows that $v$ is a coincidence point of the pair $(A, S)$.

As $z \in T(X)$, there exists a point $\vartheta \in X$ such that $T \vartheta=z$. We show that $B \vartheta=T \vartheta$. Using inequality (3.1) with $x=v, y=\vartheta$, we get

$$
\mathfrak{g}\left(F_{A v, B \vartheta}(t)\right)
$$




$$
\leq \phi\left(\max \left\{\begin{array}{c}
\mathfrak{g}\left(F_{S v, T \vartheta}(t)\right), \mathfrak{g}\left(F_{S v, A v}(t)\right), \mathfrak{g}\left(F_{T \vartheta, B \vartheta}(t)\right) \\
\frac{1}{2}\left[\mathfrak{g}\left(F_{A v, S v}(t)\right)+\mathfrak{g}\left(F_{B \vartheta, T \vartheta}(t)\right)\right], \frac{1}{2}\left[\mathfrak{g}\left(F_{A v, S v}(t)\right)+\mathfrak{g}\left(F_{S v, T \vartheta}(t)\right)\right], \\
\frac{1}{2}\left[\mathfrak{g}\left(F_{B \vartheta, T \vartheta}(t)\right)+\mathfrak{g}\left(F_{S v, T \vartheta}(t)\right)\right], \frac{1}{2}\left(\mathfrak{g}\left(F_{S v, B \vartheta}(t)\right)+\mathfrak{g}\left(F_{T \vartheta, A v}(t)\right)\right)
\end{array}\right\}\right)
$$

that is,

$$
\begin{aligned}
& \mathfrak{g}\left(F_{z, B \vartheta}(t)\right) \\
\leq & \phi\left(\max \left\{\begin{array}{c}
\mathfrak{g}\left(F_{z, z}(t)\right), \mathfrak{g}\left(F_{z, z}(t)\right), \mathfrak{g}\left(F_{z, B \vartheta}(t)\right), \frac{1}{2}\left[\mathfrak{g}\left(F_{z, z}(t)\right)+\mathfrak{g}\left(F_{B \vartheta, z}(t)\right)\right], \\
\frac{1}{2}\left[\mathfrak{g}\left(F_{z, z}(t)\right)+\mathfrak{g}\left(F_{z, z}(t)\right)\right], \\
\frac{1}{2}\left[\mathfrak{g}\left(F_{B \vartheta, z}(t)\right)+\mathfrak{g}\left(F_{z, z}(t)\right)\right], \frac{1}{2}\left(\mathfrak{g}\left(F_{z, B \vartheta}(t)\right)+\mathfrak{g}\left(F_{z, z}(t)\right)\right)
\end{array}\right\}\right) \\
= & \phi\left(\max \left\{\mathfrak{g}(1), \mathfrak{g}(1), \mathfrak{g}\left(F_{z, B v}(t)\right), \frac{1}{2} \mathfrak{g}\left(F_{B \vartheta, z}(t)\right), \mathfrak{g}(1), \frac{1}{2} \mathfrak{g}\left(F_{B \vartheta, z}(t)\right), \frac{1}{2} \mathfrak{g}\left(F_{z, B v}(t)\right)\right\}\right) \\
= & \phi\left(\max \left\{0,0, \mathfrak{g}\left(F_{z, B v}(t)\right), \frac{1}{2} \mathfrak{g}\left(F_{B \vartheta, z}(t)\right), 0, \frac{1}{2} \mathfrak{g}\left(F_{B \vartheta, z}(t)\right), \frac{1}{2} \mathfrak{g}\left(F_{z, B v}(t)\right)\right\}\right) \\
= & \phi\left(\mathfrak{g}\left(F_{z, B v}(t)\right)\right) .
\end{aligned}
$$

Hence, by Lemma 3.1, we have $B \vartheta=T \vartheta=z$, which shows that $\vartheta$ is a coincidence point of the pair $(B, T)$.

In the case when the pair $(A, S)$ is weakly compatible, $A v=S v$, imply that $A z=A S v=S A v=S z$. Now, we show that $z$ is a common fixed point of the pair $(A, S)$. Putting $x=z$ and $y=\vartheta$ in inequality (3.1), we have

$$
\begin{aligned}
& \mathfrak{g}\left(F_{A z, B v}(t)\right) \\
\leq & \phi\left(\max \left\{\begin{array}{c}
\mathfrak{g}\left(F_{S z, T v}(t)\right), \mathfrak{g}\left(F_{S z, A z}(t)\right), \mathfrak{g}\left(F_{T v, B v}(t)\right), \\
\frac{1}{2}\left[\mathfrak{g}\left(F_{A z, S z}(t)\right)+\mathfrak{g}\left(F_{B \vartheta, T \vartheta}(t)\right)\right], \frac{1}{2}\left[\mathfrak{g}\left(F_{A z, S z}(t)\right)+\mathfrak{g}\left(F_{S z, T \vartheta}(t)\right)\right], \\
\frac{1}{2}\left[\mathfrak{g}\left(F_{B \vartheta, T \vartheta}(t)\right)+\mathfrak{g}\left(F_{S z, T \vartheta}(t)\right)\right], \frac{1}{2}\left(\mathfrak{g}\left(F_{S z, B v}(t)\right)+\mathfrak{g}\left(F_{T v, A z}(t)\right)\right)
\end{array}\right\}\right)
\end{aligned}
$$

implying that

$$
\begin{aligned}
& \mathfrak{g}\left(F_{A z, z}(t)\right) \\
\leq & \phi\left(\max \left\{\begin{array}{c}
\mathfrak{g}\left(F_{A z, z}(t)\right), \mathfrak{g}\left(F_{A z, A z}(t)\right), \mathfrak{g}\left(F_{z, z}(t)\right), \\
\frac{1}{2}\left[\mathfrak{g}\left(F_{A z, A z}(t)\right)+\mathfrak{g}\left(F_{z, z}(t)\right)\right], \frac{1}{2}\left[\mathfrak{g}\left(F_{A z, A z}(t)\right)+\mathfrak{g}\left(F_{A z, z}(t)\right)\right], \quad \\
\frac{1}{2}\left[\mathfrak{g}\left(F_{z, z}(t)\right)+\mathfrak{g}\left(F_{A z, z}(t)\right)\right], \frac{1}{2}\left(\mathfrak{g}\left(F_{A z, z}(t)\right)+\mathfrak{g}\left(F_{z, A z}(t)\right)\right)
\end{array}\right\}\right) \\
= & \phi\left(\max \left\{\mathfrak{g}\left(F_{A z, z}(t)\right), \mathfrak{g}(1), \mathfrak{g}(1), \mathfrak{g}(1), \frac{1}{2} \mathfrak{g}\left(F_{A z, z}(t)\right), \frac{1}{2} \mathfrak{g}\left(F_{A z, z}(t)\right), \mathfrak{g}\left(F_{A z, z}(t)\right)\right\}\right) \\
= & \phi\left(\max \left\{\mathfrak{g}\left(F_{A z, z}(t)\right), 0,0,0, \frac{1}{2} \mathfrak{g}\left(F_{A z, z}(t)\right), \frac{1}{2} \mathfrak{g}\left(F_{A z, z}(t)\right), \mathfrak{g}\left(F_{A z, z}(t)\right)\right\}\right) \\
= & \phi\left(\mathfrak{g}\left(F_{A z, z}(t)\right)\right) .
\end{aligned}
$$

Again making use of Lemma 3.1, we have $A z=z=S z$ which shows that $z$ is a common fixed point of the pair $(A, S)$.

Again, when the pair $(B, T)$ is weakly compatible, then $B \vartheta=T \vartheta$ implies that $B z=B T \vartheta=T B \vartheta=T z$. Putting $x=v, y=z$ in inequality (3.1), we have

$$
\begin{aligned}
& \mathfrak{g}\left(F_{A v, B z}(t)\right) \\
\leq & \phi\left(\max \left\{\begin{array}{c}
\mathfrak{g}\left(F_{S v, T z}(t)\right), \mathfrak{g}\left(F_{S v, A v}(t)\right), \mathfrak{g}\left(F_{T z, B z}(t)\right), \\
\frac{1}{2}\left[\mathfrak{g}\left(F_{A z, S z}(t)\right)+\mathfrak{g}\left(F_{B \vartheta, T \vartheta}(t)\right)\right], \frac{1}{2}\left[\mathfrak{g}\left(F_{A z, S z}(t)\right)+\mathfrak{g}\left(F_{S z, T \vartheta}(t)\right)\right], \\
\frac{1}{2}\left[\mathfrak{g}\left(F_{B \vartheta, T \vartheta}(t)\right)+\mathfrak{g}\left(F_{S z, T \vartheta}(t)\right)\right], \frac{1}{2}\left(\mathfrak{g}\left(F_{S v, B z}(t)\right)+\mathfrak{g}\left(F_{T z, A v}(t)\right)\right)
\end{array}\right\}\right),
\end{aligned}
$$


that is,

$$
\begin{aligned}
& \mathfrak{g}\left(F_{z, B z}(t)\right) \\
& \leq \phi\left(\max \left\{\begin{array}{c}
\mathfrak{g}\left(F_{z, B z}(t)\right), \mathfrak{g}\left(F_{z, z}(t)\right), \mathfrak{g}\left(F_{B z, B z}(t)\right), \\
\frac{1}{2}\left[\mathfrak{g}\left(F_{A z, A z}(t)\right)+\mathfrak{g}\left(F_{z, z}(t)\right)\right], \frac{1}{2}\left[\mathfrak{g}\left(F_{A z, A z}(t)\right)+\mathfrak{g}\left(F_{S z, z}(t)\right)\right], \\
\frac{1}{2}\left[\mathfrak{g}\left(F_{z, z}(t)\right)+\mathfrak{g}\left(F_{A z, z}(t)\right)\right], \frac{1}{2}\left(\mathfrak{g}\left(F_{z, B z}(t)\right)+\mathfrak{g}\left(F_{B z, z}(t)\right)\right)
\end{array}\right\}\right) \\
& =\phi\left(\max \left\{\begin{array}{c}
\mathfrak{g}\left(F_{z, B z}(t)\right), \mathfrak{g}(1), \mathfrak{g}(1), \frac{1}{2}[\mathfrak{g}(1)+\mathfrak{g}(1)], \frac{1}{2}\left[\mathfrak{g}(1)+\mathfrak{g}\left(F_{A z, z}(t)\right)\right], \\
\frac{1}{2}[\mathfrak{g}(1)+\mathfrak{g}(1)], \frac{1}{2}\left(\mathfrak{g}\left(F_{z, B z}(t)\right)+\mathfrak{g}\left(F_{B z, z}(t)\right)\right)
\end{array}\right\}\right) \\
& =\phi\left(\max \left\{\mathfrak{g}\left(F_{z, B z}(t)\right), 0,0,0, \frac{1}{2} \mathfrak{g}\left(F_{A z, z}(t)\right), 0, \mathfrak{g}\left(F_{B z, z}(t)\right)\right\}\right) \\
& =\phi\left(\mathfrak{g}\left(F_{z, B z}(t)\right)\right) \text {. }
\end{aligned}
$$

Using Lemma 3.1, we have $B z=z=T z$ which shows that $z$ is a common fixed point of the pair $(B, T)$ and in all $z$ is a common fixed point of the pairs $(A, S)$ and $(B, T)$. The uniqueness of common fixed point is an easy consequence of inequality (3.1) in view of Lemma 3.1. This concludes the proof.

The following proposition will help us to get further results.

Proposition 3.3. Let $A, B, S$ and $T$ be four self mappings of an N. A. Menger PM-space $(X, \mathcal{F}, \mathcal{T})$, where $\mathcal{T}$ is a continuous t-norm. Suppose that

(1) the pair $(A, S)$ satisfies the $\left(C L R_{S}\right)$ property (or the pair $(B, T)$ satisfies the $\left(C L R_{T}\right)$ property),

(2) $A(X) \subset T(X)($ or $B(X) \subset S(X))$,

(3) $T(X)($ or $S(X))$ is a closed subset of $X$,

(4) $\left\{B y_{n}\right\}$ converges for every sequence $\left\{y_{n}\right\}$ in $X$ whenever $\left\{T y_{n}\right\}$ converges (or $\left\{A x_{n}\right\}$ converges for every sequence $\left\{x_{n}\right\}$ in $X$ whenever $\left\{S x_{n}\right\}$ converges),

(5) the mappings $A, B, S$ and $T$ satisfy inequality (3.1) of Theorem 3.2. Then the pairs $(A, S)$ and $(B, T)$ enjoy the $\left(C L R_{S T}\right)$ property.

Proof. If the pair $(A, S)$ satisfies the $\left(C L R_{S}\right)$ property, then there exists a sequence $\left\{x_{n}\right\}$ in $X$ such that

$$
\lim _{n \rightarrow \infty} A x_{n}=\lim _{n \rightarrow \infty} S x_{n}=z,
$$

where $z \in S(X)$. Since $A(X) \subset T(X)$, hence for each $\left\{x_{n}\right\} \subset X$ there corresponds a sequence $\left\{y_{n}\right\} \subset X$ such that $A x_{n}=T y_{n}$. Therefore, due to closedness of $T(X)$,

$$
\lim _{n \rightarrow \infty} T y_{n}=\lim _{n \rightarrow \infty} A x_{n}=z,
$$

where $z \in S(X) \cap T(X)$. Thus in all, we have $A x_{n} \rightarrow z, S x_{n} \rightarrow z$ and $T y_{n} \rightarrow z$ as $n \rightarrow \infty$. By (4), the sequence $\left\{B y_{n}\right\}$ converges and we just need to show 
that $B y_{n} \rightarrow z$ as $n \rightarrow \infty$. Putting $x=x_{n}, y=y_{n}$ in inequality (3.1), we get

$$
\begin{aligned}
& \mathfrak{g}\left(F_{A x_{n}, B y_{n}}(t)\right) \\
\leq & \phi\left(\max \left\{\begin{array}{c}
\mathfrak{g}\left(F_{S x_{n}, T y_{n}}(t)\right), \mathfrak{g}\left(F_{S x_{n}, A x_{n}}(t)\right), \mathfrak{g}\left(F_{T y_{n}, B y_{n}}(t)\right), \\
\frac{1}{2}\left[\mathfrak{g}\left(F_{A x_{n}, S x_{n}}(t)\right)+\mathfrak{g}\left(F_{B y_{n}, T y_{n}}(t)\right)\right], \frac{1}{2}\left[\mathfrak{g}\left(F_{A x_{n}, S x_{n}}(t)\right)+\mathfrak{g}\left(F_{S x_{n}, T y_{n}}(t)\right)\right], \\
\frac{1}{2}\left[\mathfrak{g}\left(F_{B y_{n}, T y_{n}}(t)\right)+\mathfrak{g}\left(F_{S x_{n}, T y_{n}}(t)\right)\right], \frac{1}{2}\left[\mathfrak{g}\left(F_{S x_{n}, B y_{n}}(t)\right)+\mathfrak{g}\left(F_{T y_{n}, A x_{n}}(t)\right)\right]
\end{array}\right\}\right) .
\end{aligned}
$$

Let $B y_{n} \rightarrow l(\neq z)$ as $n \rightarrow \infty$. Then, passing to the limit as $n \rightarrow \infty$, we get

$$
\begin{aligned}
& \mathfrak{g}\left(F_{z, l}(t)\right) \\
\leq & \phi\left(\max \left\{\begin{array}{c}
\mathfrak{g}\left(F_{z, z}(t)\right), \mathfrak{g}\left(F_{z, z}(t)\right), \mathfrak{g}\left(F_{z, l}(t)\right), \\
\frac{1}{2}\left[\mathfrak{g}\left(F_{z, z}(t)\right)+\mathfrak{g}\left(F_{l, z}(t)\right)\right], \frac{1}{2}\left[\mathfrak{g}\left(F_{z, z}(t)\right)+\mathfrak{g}\left(F_{z, z}(t)\right)\right], \\
\frac{1}{2}\left[\mathfrak{g}\left(F_{l, z}(t)\right)+\mathfrak{g}\left(F_{z, z}(t)\right)\right], \frac{1}{2}\left(\mathfrak{g}\left(F_{z, l}(t)\right)+\mathfrak{g}\left(F_{z, z}(t)\right)\right)
\end{array}\right\}\right), \\
= & \phi\left(\max \left\{\begin{array}{c}
\mathfrak{g}(1), \mathfrak{g}(1), \mathfrak{g}\left(F_{z, l}(t)\right), \frac{1}{2}\left[\mathfrak{g}(1)+\mathfrak{g}\left(F_{l, z}(t)\right)\right], \frac{1}{2}[\mathfrak{g}(1)+\mathfrak{g}(1)], \\
\frac{1}{2}\left[\mathfrak{g}\left(F_{l, z}(t)\right)+\mathfrak{g}(1)\right], \frac{1}{2}\left(\mathfrak{g}\left(F_{z, l}(t)\right)+\mathfrak{g}(1)\right)
\end{array}\right\}\right) \\
= & \phi\left(\max \left\{0,0, \mathfrak{g}\left(F_{z, l}(t)\right), \frac{1}{2}\left(\mathfrak{g}\left(F_{z, l}(t)\right), 0, \frac{1}{2}\left(\mathfrak{g}\left(F_{z, l}(t)\right)\right), \frac{1}{2}\left(\mathfrak{g}\left(F_{z, l}(t)\right)\right)\right\}\right)\right. \\
= & \phi\left(\mathfrak{g}\left(F_{z, l}(t)\right)\right) .
\end{aligned}
$$

So, by Lemma 3.1 , we have $z=l$. Hence the pairs $(A, S)$ and $(B, T)$ share the $\left(C L R_{S T}\right)$ property.

The converse of Proposition 3.3 is not true. For a counterexample see $[17$, Example 3.5].

Theorem 3.4. Let $A, B, S$ and $T$ be four self mappings of an N. A. Menger PM-space $(X, \mathcal{F}, \mathcal{T})$, where $\mathcal{T}$ is a continuous t-norm, satisfying all the hypotheses of Proposition 3.3. Then $A, B, S$ and $T$ have a unique common fixed point provided that both pairs $(A, S)$ and $(B, T)$ are weakly compatible.

Proof. This follows by combining Theorem 3.2 with Proposition 3.3.

Obviously, if the pairs $(A, S)$ and $(B, T)$ satisfy the common property (E.A), and, at the same time, $S(X)$ and $T(X)$ are closed subsets of $X$, then the pairs $(A, S)$ and $(B, T)$ share the $\left(C L R_{S T}\right)$ property. Hence, we have the following variant of Theorem 3.2.

Theorem 3.5. Let $A, B, S$ and $T$ be four self mappings of an N. A. Menger PM-space $(X, \mathcal{F}, \mathcal{T})$, where $\mathcal{T}$ is a continuous t-norm, satisfying inequality (3.1) and the following hypotheses hold:

(1) the pairs $(A, S)$ and $(B, T)$ satisfy the common property $(E . A)$;

(2) $S(Y)$ and $T(Y)$ are closed subsets of $X$.

Then $(A, S)$ and $(B, T)$ have a coincidence point each. Moreover, $A, B, S$ and $T$ have a unique common fixed point provided both pairs $(A, S)$ and $(B, T)$ are weakly compatible.

Next, we state two more variants of our results, which can be proved on the lines of the proofs of Theorems 3.4 and 3.5. 
Corollary 3.6. The conclusions of Theorem 3.5 remain true if condition (2) is replaced by the following:

$\left(2^{\prime}\right) \overline{A(X)} \subset T(X)$ and $\overline{B(X)} \subset S(X)$,

where $\overline{A(X)}$ and $\overline{B(X)}$ denote the closure of ranges of the mappings $A$ and $B$.

Corollary 3.7. The conclusions of Theorem 3.5 remain true if the condition (2) is replaced by the following:

$\left(2^{\prime \prime}\right) A(X)$ and $B(X)$ are closed subsets of $X$, and $A(X) \subset T(X), B(X) \subset$ $S(X)$.

By choosing $A, B, S$ and $T$ suitably in Theorem 3.2 , we can deduce some corollaries for a pair as well as for a triple of self mappings. Since the formulations of these results are similar to those in $[15,17]$, we omit the details here. Now we utilize this notion for six self mappings in an N. A. Menger PM-space.

Theorem 3.8. Let $A, B, R, S, H$ and $T$ be six self mappings of an N.A. Menger PM-space $(X, \mathcal{F}, \mathcal{T})$, where $\mathcal{T}$ is a continuous t-norm. Suppose that

(1) the pairs $(A, S R)$ and $(B, T H)$ satisfy the $\left(C L R_{(S R)(T H)}\right)$ property,

$$
\begin{aligned}
& \mathfrak{g}\left(F_{A x, B y}(t)\right) \\
\leq & \phi\left(\max \left\{\begin{array}{c}
\mathfrak{g}\left(F_{S R x, T H y}(t)\right), \mathfrak{g}\left(F_{S R x, A x}(t)\right), \mathfrak{g}\left(F_{T H y, B y}(t)\right), \\
\frac{1}{2}\left[\mathfrak{g}\left(F_{A x, S R x}(t)\right)+\mathfrak{g}\left(F_{B y, T H y}(t)\right)\right], \frac{1}{2}\left[\mathfrak{g}\left(F_{A x, S R x}(t)\right)+\mathfrak{g}\left(F_{S R x, T H y}(t)\right)\right], \\
\frac{1}{2}\left[\mathfrak{g}\left(F_{B y, T H y}(t)\right)+\mathfrak{g}\left(F_{S R x, T H y}(t)\right)\right], \frac{1}{2}\left(\mathfrak{g}\left(F_{S R x, B y}(t)\right)+\mathfrak{g}\left(F_{T H y, A x}(t)\right)\right)
\end{array}\right\}\right) \\
& \text { for all } x, y \in X, t>0, \text { where } \mathfrak{g} \in \Omega \text { and } \phi \in \Phi .
\end{aligned}
$$

Then $(A, S R)$ and $(B, T H)$ have a coincidence point each. Moreover, $A, B, H$, $R, S$ and $T$ have a unique common fixed point provided $A S=S A, A R=R A$, $S R=R S, B T=T B, B H=H B$ and $T H=H T$.

Proof. By Theorem 3.2, A, B, SR and TH have a unique common fixed point $z$ in $X$. We show that $z$ is a unique common fixed point of the self mappings $A, B, R, S, H$ and $T$. Putting $x=R z$ and $y=z$ in inequality (3.2), we have

$$
\begin{aligned}
& \mathfrak{g}\left(F_{A(R z), B z}(t)\right) \\
\leq & \left(\max \left\{\begin{array}{c}
\mathfrak{g}\left(F_{S R(R z), T H z}(t)\right), \mathfrak{g}\left(F_{S R(R z), A(R z)}(t)\right), \mathfrak{g}\left(F_{T H z, B z}(t)\right), \\
\frac{1}{2}\left[\mathfrak{g}\left(F_{A(R z), S R(R z)}(t)\right)+\mathfrak{g}\left(F_{B z, T H z}(t)\right)\right], \\
\frac{1}{2}\left[\mathfrak{g}\left(F_{A(R z), S R(R z)}(t)\right)+\mathfrak{g}\left(F_{S R(R z), T H z}(t)\right)\right], \\
\frac{1}{2}\left[\mathfrak{g}\left(F_{B z, T H z}(t)\right)+\mathfrak{g}\left(F_{S R(R z), T H z}(t)\right)\right], \\
\frac{1}{2}\left(\mathfrak{g}\left(F_{S R(R z), B z}(t)\right)+\mathfrak{g}\left(F_{T H z, A(R z)}(t)\right)\right)
\end{array}\right\}\right), \\
\leq & \phi\left(\operatorname{grax}\left\{\begin{array}{c}
\mathfrak{g}\left(F_{R z, z}(t)\right), \mathfrak{g}\left(F_{R z, R z}(t)\right), \mathfrak{g}\left(F_{z, z}(t)\right), \frac{1}{2}\left[\mathfrak{g}\left(F_{R z, R z}(t)\right)+\mathfrak{g}\left(F_{z, z}(t)\right)\right], \\
\frac{1}{2}\left[\mathfrak{g}\left(F_{R z, R z}(t)\right)+\mathfrak{g}\left(F_{R z, z}(t)\right)\right], \frac{1}{2}\left[\mathfrak{g}\left(F_{z, z}(t)\right)+\mathfrak{g}\left(F_{R z, z}(t)\right)\right], \\
\frac{1}{2}\left(\mathfrak{g}\left(F_{R z, z}(t)\right)+\mathfrak{g}\left(F_{z, R z}(t)\right)\right)
\end{array}\right\}\right)
\end{aligned}
$$




$$
\begin{aligned}
& =\phi\left(\max \left\{\begin{array}{c}
\left.\mathfrak{g}\left(F_{R z, z}(t)\right), \mathfrak{g}(1), \mathfrak{g}(1), \mathfrak{g}(1)\right), \frac{1}{2} \mathfrak{g}\left(F_{R z, z}(t)\right), \frac{1}{2} \mathfrak{g}\left(F_{R z, z}(t)\right), \\
\frac{1}{2}\left(\mathfrak{g}\left(F_{R z, z}(t)\right)+\mathfrak{g}\left(F_{z, R z}(t)\right)\right)
\end{array}\right\}\right) \\
& =\phi\left(\max \left\{\mathfrak{g}\left(F_{R z, z}(t)\right), 0,0,0, \frac{1}{2} \mathfrak{g}\left(F_{R z, z}(t)\right), \frac{1}{2} \mathfrak{g}\left(F_{R z, z}(t)\right), \mathfrak{g}\left(F_{R z, z}(t)\right)\right\}\right) \\
& =\phi\left(\mathfrak{g}\left(F_{R z, z}(t)\right)\right) .
\end{aligned}
$$

Using Lemma 3.1, we have $z=R z$. Hence $S z=S(R z)=z$. Therefore we have $z=A z=S z=R z$. Now we assert that $z$ is a common fixed point of $B, T$ and $H$. To accomplish this, we use inequality (3.2) with $x=z, y=H z$, and get

$$
\begin{aligned}
& \mathfrak{g}\left(F_{A z, B(H z)}(t)\right) \\
\leq & \left(\max \left\{\begin{array}{c}
\mathfrak{g}\left(F_{S R z, T H(H z)}(t)\right), \mathfrak{g}\left(F_{S R z, A z}(t)\right), \mathfrak{g}\left(F_{T H(H z), B(H z)}(t)\right), \\
\frac{1}{2}\left[\mathfrak{g}\left(F_{A z, S R z}(t)\right)+\mathfrak{g}\left(F_{B(H z), T H(H z)}(t)\right)\right], \\
\frac{1}{2}\left[\mathfrak{g}\left(F_{A(H z), S R(H z)}(t)\right)+\mathfrak{g}\left(F_{S R z, T H(H z)}(t)\right)\right] \\
\frac{1}{2}\left[\mathfrak{g}\left(F_{B(H z), T H(H z)}(t)\right)+\mathfrak{g}\left(F_{S R z, T H(H z)}(t)\right)\right], \\
\frac{1}{2}\left(\mathfrak{g}\left(F_{S R z, B(H z)}(t)\right)+\mathfrak{g}\left(F_{T H(H z), A z}(t)\right)\right)
\end{array}\right\}\right),
\end{aligned}
$$

that is,

$$
\begin{aligned}
& \mathfrak{g}\left(F_{z, H z}(t)\right) \\
\leq & \phi\left(\max \left\{\begin{array}{c}
\mathfrak{g}\left(F_{z, H z}(t)\right), \mathfrak{g}\left(F_{z, z}(t)\right), \mathfrak{g}\left(F_{H z, H z}(t)\right), \\
\frac{1}{2}\left[\mathfrak{g}\left(F_{z, z}(t)\right)+\mathfrak{g}\left(F_{H z, H z}(t)\right)\right], \frac{1}{2}\left[\mathfrak{g}\left(F_{H z, H z}(t)\right)+\mathfrak{g}\left(F_{z, H z}(t)\right)\right], \\
\frac{1}{2}\left[\mathfrak{g}\left(F_{H z, H z}(t)\right)+\mathfrak{g}\left(F_{z, H z}(t)\right], \frac{1}{2}\left(\mathfrak{g}\left(F_{z, H z}(t)\right)+\mathfrak{g}\left(F_{H z, z}(t)\right)\right)\right.
\end{array}\right\}\right) \\
= & \phi\left(\max \left\{\mathfrak{g}\left(F_{z, H z}(t)\right), \mathfrak{g}(1), \mathfrak{g}(1), \mathfrak{g}(1), \frac{1}{2} \mathfrak{g}\left(F_{z, H z}(t)\right), \frac{1}{2} \mathfrak{g}\left(F_{z, H z}(t)\right), \mathfrak{g}\left(F_{H z, z}(t)\right)\right\}\right) \\
= & \phi\left(\max \left\{\mathfrak{g}\left(F_{z, H z}(t)\right), 0,0,0, \frac{1}{2} \mathfrak{g}\left(F_{z, H z}(t)\right), \frac{1}{2} \mathfrak{g}\left(F_{z, H z}(t)\right), \mathfrak{g}\left(F_{H z, z}(t)\right)\right\}\right) \\
= & \phi\left(\mathfrak{g}\left(F_{z, H z}(t)\right)\right) .
\end{aligned}
$$

Thus, by Lemma 3.1, we have $z=H z$. Hence $T z=T(H z)=z$. Therefore $z$ is a common fixed point of self mappings $A, B, R, S, H$ and $T$. Uniqueness of common fixed point is an easy consequence of inequality (3.2).

In view of Theorem 3.4, we can derive a fixed point theorem for four finite families of self mappings.

Corollary 3.9. Let $\left\{A_{i}\right\}_{i=1}^{m},\left\{B_{r}\right\}_{r=1}^{n},\left\{S_{k}\right\}_{k=1}^{p}$ and $\left\{T_{h}\right\}_{h=1}^{q}$ be four finite families of self mappings of an N. A. Menger PM-space $(X, \mathcal{F}, \mathcal{T})$, where $\mathcal{T}$ is a continuous t-norm, with $A=A_{1} A_{2} \cdots A_{m}, B=B_{1} B_{2} \cdots B_{n}, S=$ $S_{1} S_{2} \cdots S_{p}$ and $T=T_{1} T_{2} \cdots T_{q}$ satisfying inequality (3.1) of Theorem 3.2 such that the pairs $(A, S)$ and $(B, T)$ share the $\left(C L R_{S T}\right)$ property. Then $\left\{A_{i}\right\}_{i=1}^{m}$, $\left\{B_{r}\right\}_{r=1}^{n},\left\{S_{k}\right\}_{k=1}^{p}$ and $\left\{T_{h}\right\}_{h=1}^{q}$ have a unique common fixed point provided the pairs of families $\left(\left\{A_{i}\right\},\left\{S_{k}\right\}\right)$ and $\left(\left\{B_{r}\right\},\left\{T_{h}\right\}\right)$ commute pairwise, where $i \in\{1,2, \ldots, m\}, k \in\{1,2, \ldots, p\}, r \in\{1,2, \ldots, n\}$ and $h \in\{1,2, \ldots, q\}$.

By setting $A_{1}=A_{2}=\cdots=A_{m}=A, B_{1}=B_{2}=\cdots=B_{p}=B, S_{1}=S_{2}=$ $\cdots=S_{n}=S$ and $T_{1}=T_{2}=\cdots=T_{q}=T$ in Corollary 3.9, we deduce the following: 
Corollary 3.10. Let $A, B, S$ and $T$ be self mappings of an N. A. Menger PM-space $(X, \mathcal{F}, \mathcal{T})$, where $\mathcal{T}$ is a continuous t-norm. Suppose that

(1) the pairs $\left(A^{m}, S^{p}\right)$ and $\left(B^{n}, T^{q}\right)$ satisfy the $\left(C L R_{S^{p}, T^{q}}\right)$ property, where $m, n, p, q$ are fixed positive integers,

$$
\begin{aligned}
& \mathfrak{g}\left(F_{A^{m} x, B^{n} y}(t)\right) \\
& \leq \phi\left(\max \left\{\begin{array}{c}
\mathfrak{g}\left(F_{S^{p} x, T^{q} y}(t)\right), \mathfrak{g}\left(F_{S^{p} x, A^{m} x}(t)\right), \mathfrak{g}\left(F_{T^{q} y, B^{n} y}(t)\right), \\
\left.\frac{1}{2}\left[\mathfrak{g}\left(F_{A^{m} x, S^{p} x}(t)\right)+\mathfrak{g}\left(F_{B^{n} y, T^{q} y}(t)\right)\right], \frac{1}{2} \mathfrak{g}\left(F_{A^{m} x, S^{p} x}(t)\right)+\mathfrak{g}\left(F_{S^{p} x, T^{q} y}(t)\right)\right], \\
\frac{1}{2}\left[\mathfrak{g}\left(F_{B^{n} y, T^{q} y}(t)\right)+\mathfrak{g}\left(F_{S^{p} x, T^{q} y}(t)\right)\right], \frac{1}{2}\left(\mathfrak{g}\left(F_{S^{p} x, B^{n} y}(t)\right)+\mathfrak{g}\left(F_{T^{q} y, A^{m} x}(t)\right)\right)
\end{array}\right\}\right) \\
& \text { for all } x, y \in X, t>0, \mathfrak{g} \in \Omega \text { where } \phi \in \Phi .
\end{aligned}
$$

Then $A, B, S$ and $T$ have a unique common fixed point provided $A S=S A$ and $B T=T B$.

Remark 3.11. The conclusions of Theorem 3.2 remain true if we replace the inequality (3.1) by any of the following (for all $x, y \in X, t>0$, where $\mathfrak{g} \in \Omega$ and $\phi$ belongs to the class $\Phi)$ :

$$
\begin{aligned}
& \mathfrak{g}\left(F_{A x, B y}(t)\right) \\
\leq & \phi\left(\max \left\{\begin{array}{c}
\mathfrak{g}\left(F_{S x, T y}(t)\right), \mathfrak{g}\left(F_{S x, A x}(t)\right), \mathfrak{g}\left(F_{T y, B y}(t)\right), \mathfrak{g}\left(F_{S x, B y}(t)\right) \\
\frac{1}{2}\left[\mathfrak{g}\left(F_{B y, T y}(t)\right)+\mathfrak{g}\left(F_{S x, T y}(t)\right)\right]
\end{array}\right\}\right),
\end{aligned}
$$

or

$$
\mathfrak{g}\left(F_{A x, B y}(t)\right) \leq \phi\left(\max \left\{\begin{array}{c}
\mathfrak{g}\left(F_{S x, T y}(t)\right), \mathfrak{g}\left(F_{S x, A x}(t)\right), \mathfrak{g}\left(F_{T y, B y}(t)\right), \\
\frac{1}{2}\left[\mathfrak{g}\left(F_{A x, S x}(t)\right)+\mathfrak{g}\left(F_{B y, T y}(t)\right)\right]
\end{array}\right\}\right),
$$

or

$$
\mathfrak{g}\left(F_{A x, B y}(t)\right) \leq \phi\left(\max \left\{\begin{array}{c}
\mathfrak{g}\left(F_{S x, T y}(t)\right), \mathfrak{g}\left(F_{S x, A x}(t)\right), \mathfrak{g}\left(F_{T y, B y}(t)\right), \\
\frac{1}{2}\left[\mathfrak{g}\left(F_{A x, S x}(t)\right)+\mathfrak{g}\left(F_{S x, T y}(t)\right)\right]
\end{array}\right\}\right),
$$

or

$$
\mathfrak{g}\left(F_{A x, B y}(t)\right) \leq \phi\left(\max \left\{\begin{array}{c}
\mathfrak{g}\left(F_{S x, T y}(t)\right), \mathfrak{g}\left(F_{S x, A x}(t)\right), \mathfrak{g}\left(F_{T y, B y}(t)\right), \\
\frac{1}{2}\left[\mathfrak{g}\left(F_{S x, B y}(t)\right)+\mathfrak{g}\left(F_{T y, A x}(t)\right)\right]
\end{array}\right\}\right) .
$$

Remark 3.12. The results similar to Theorem 3.4, Theorem 3.5, Theorem 3.8, Corollary 3.9 and Corollary 3.10 can also be outlined in view of inequalities (3.3)-(3.6).

Remark 3.13. Our Theorems 3.2, 3.4, 3.5 and 3.8, as well as Corollaries 3.6, 3.7, 3.9 and 3.10 extend and improve the following:

(1) the results of Chauhan and Vujakovic [8] in the sense of using more general contractive condition and relaxing closedness of mappings.

(2) the results of Chauhan et al. [7] in the sense of using more general contractive condition and relaxing closedness of mappings in non-integral version. 
(3) the results of Khan and Sumitra [26, Theorem 2, Corollary 1], Singh et al. [32, Theorem 3.1, Corollary 3.3], Singh et al. [33, Theorem 3.1, Corollary 3.1] and Rao and Ramudu [29, Theorem 14].

\section{Illustrative examples}

Now we furnish examples demonstrating the validity of the hypotheses and degree of generality of our results over some recently established results.

Example 4.1. Let $(X, \mathcal{F}, \mathcal{T})$ be an N. A. Menger PM-space, where $X=$ $[2,11)$ and metric $d$ is defined as in condition (2) of Remark 2.3. Consider the mappings $A, B, S, T: X \rightarrow X$ given by

$$
\begin{array}{cc}
A x= \begin{cases}2, & \text { if } x \in\{2\} \cup(5,11), \\
8, & \text { if } x \in(2,5] ;\end{cases} & B x= \begin{cases}2, & \text { if } x \in\{2\} \cup(5,11), \\
4, & \text { if } x \in(2,5] ;\end{cases} \\
S x= \begin{cases}2, & \text { if } x=2, \\
9, & \text { if } x \in(2,5], \\
\frac{x+1}{3}, & \text { if } x \in(5,11) ;\end{cases} & T x= \begin{cases}2, & \text { if } x=2, \\
6, & \text { if } x \in(2,5], \\
x-3, & \text { if } x \in(5,11) .\end{cases}
\end{array}
$$

Then we have $A(X)=\{2,8\} \nsubseteq[2,8)=T(X)$ and $B(X)=\{2,4\} \nsubseteq[2,4) \cup$ $\{9\}=S(X)$, moreover $S(X)$ and $T(X)$ are not closed subsets of $X$.

The pairs $(A, S)$ and $(B, T)$ satisfy the $\left(C L R_{S T}\right)$ property. Indeed, taking two sequences, $\left\{x_{n}\right\}=\left\{5+\frac{1}{n}\right\}_{n \in \mathbb{N}},\left\{y_{n}\right\}=\{2\}$, we have

$$
\lim _{n \rightarrow \infty} A x_{n}=\lim _{n \rightarrow \infty} S x_{n}=\lim _{n \rightarrow \infty} B y_{n}=\lim _{n \rightarrow \infty} T y_{n}=2 \in S(X) \cap T(X) .
$$

Now, define a function $\varphi:[0,+\infty) \rightarrow[0,+\infty)$ by

$$
\varphi(t)=h t, \text { with } \frac{6}{7}<h<1, \text { for all } t \geq 0 .
$$

Clearly, $\varphi \in \Phi$. By a routine calculation, one can check that the inequality (3.1) is satisfied for all $x, y \in X$. Thus, all the conditions of Theorem 3.2 are satisfied, and 2 is a unique common fixed point of the pairs $(A, S)$ and $(B, T)$. Note that all the involved mappings are discontinuous at their unique common fixed point.

In the following illustration the importance of weakly compatible assumption for validity of the result is shown.

Example 4.2. Let $(X, \mathcal{F}, \mathcal{T})$ be an N. A. Menger PM-space, where $X=$ $[0,+\infty)$ and the metric $d$ is defined as in condition (2) of Remark 2.3. Consider the mappings $A, B, S, T: X \rightarrow X$ given by

$$
A x=B x=x+3 \text { and } S x=T x=2(1+x) .
$$

Then the pairs $(A, S),(B, T)$ satisfy the $\left(C L R_{S T}\right)$ property. Indeed, consider two sequences, $\left\{x_{n}\right\}=\left\{1+\frac{1}{n}\right\}_{n \in \mathbb{N}},\left\{y_{n}\right\}=\left\{1-\frac{1}{n}\right\}_{n \in \mathbb{N}}$. Then

$$
\lim _{n \rightarrow \infty} A x_{n}=\lim _{n \rightarrow \infty} S x_{n}=\lim _{n \rightarrow \infty} B y_{n}=\lim _{n \rightarrow \infty} T y_{n}=4,
$$


where $4 \in S(X) \cap T(X)$.

By a routine calculation, taking $\varphi(t)=h t$ with a suitable value of $h$, one can check that inequality (3.1) is satisfied. Thus, all the conditions of the first part of Theorem 3.2 are satisfied. It can be noted that, indeed, 1 is a coincidence point of $(A, S)$, as well as of $(B, T)$. However, these pairs are not weakly compatible and there is no common fixed point of the pairs $(A, S)$ and $(B, T)$.

Theorem 3.4 cannot be applied in the case of mappings from Example 4.1 since conditions (2) and (3) of Proposition 3.3 are not fulfilled. The following example shows the situation when Theorem 3.4 can be used.

Example 4.3. In the setting of Example 4.1, replace the mappings $S$ and $T$ by the following, besides retaining the rest:

$$
S x=\left\{\begin{array}{ll}
2, & \text { if } x=2, \\
5, & \text { if } x \in(2,5], \\
\frac{x-1}{2}, & \text { if } x \in(5,11) ;
\end{array} \quad T x= \begin{cases}2, & \text { if } x=2, \\
8, & \text { if } x \in(2,5], \\
x-3, & \text { if } x \in(5,11) .\end{cases}\right.
$$

Then $A(X)=\{2,8\} \subset[2,8]=T(X)$ and $B(X)=\{2,4\} \subset[2,5]=S(X)$ hold; now $S(X)$ and $T(X)$ are closed subsets of $X$. Thus, all the conditions of Theorem 3.4 are satisfied, and 2 is a unique common fixed point of the pairs $(A, S)$ and $(B, T)$.

Now we furnish an example demonstrating that the condition (3.1) of Theorem 3.2 is only sufficient and not necessary.

Example 4.4. Let $(X, \mathcal{F}, \mathcal{T})$ be an N. A. Menger PM-space, where $X=$ $[2,20]$ and metric $d$ be defined as in condition (2) of Remark 2.3. Consider the mappings $A, B, S, T: X \rightarrow X$ given by

$$
A x=B x=\left\{\begin{array}{ll}
2 & \text { if } x=2, \\
7 & \text { if } 2<x \leq 5, \\
2, & \text { if } 5<x \leq 20,
\end{array} \quad S x=T x= \begin{cases}2 & \text { if } x=2, \\
7 & \text { if } 2<x \leq 5, \\
\frac{x+1}{3} & \text { if } 5<x \leq 20 .\end{cases}\right.
$$

Then the pairs $(A, S)$ and $(B, T)$ satisfy all the conditions of Theorem 3.2, except the inequality (3.1) (take, e.g., $x \in(2,5]$ and $y=2$ ). However, these four mappings have a coincidence at $x=2$ which also remains their common fixed point. This confirms that condition (3.1) of Theorem 3.2 is sufficient and not necessary.

Our last example highlights the non-closedness of ranges of $S$ and $T$ in $X$ in Corollaries 3.6 and 3.7. 
Example 4.5. In the setting of Example 4.1, replace the mappings $S$ and $T$ by the following, besides retaining the rest:

$$
S x=T x= \begin{cases}2 & \text { if } x=2, \\ 10 & \text { if } x \in(2,5], \\ \frac{7 x-23}{6} & \text { if } x \in(5,11) .\end{cases}
$$

Then $A(X)=\{2,8\} \subset[2,9) \cup\{10\}=T(X)$ and $B(X)=\{2,4\} \subset[2,9) \cup\{10\}=$ $S(X)$. Now, $S(X)$ and $T(X)$ are not closed subspaces of $X$, but condition $\left(2^{\prime}\right)$, resp. $\left(2^{\prime \prime}\right)$ of Corollary 3.6, resp. 3.7 is satisfied. Again, 2 is a unique common fixed point of $A, B, S$ and $T$.

\section{An application to a multistage process}

In this section, we study, along with $[2,3,16,39]$ and some other papers, the existence and uniqueness of solutions for a certain system of functional equations arising in dynamic programming. We consider the following system of functional equations, to which a multistage process can be reduced

$$
q(x)=\sup _{y \in D}\left\{f(x, y)+G_{i}(x, y, q(\tau(x, y)))\right\}, \quad x \in W, \quad i \in\{1,2,3,4\},
$$

where $U$ and $V$ are Banach spaces, $W \subseteq U$ is a state space, $D \subseteq V$ is a decision space, while $\tau: W \times D \rightarrow W, f: W \times D \rightarrow \mathbb{R}, G_{i}: W \times \bar{D} \times \mathbb{R} \rightarrow \mathbb{R}$ are mappings, $i \in\{1,2,3,4\}$.

Denote by $X$ the set of all bounded real-valued functions on $W$ and, for $h \in X$, define $\|h\|=\sup _{x \in W}|h(x)|$. Clearly, $(X,\|\cdot\|)$ is a Banach space, and the convergence in this space is uniform. Therefore, if $\left\{h_{n}\right\}$ is a Cauchy sequence in $X$, then it converges uniformly to a function $h^{*} \in X$. The respective metric will be denoted by $d$.

Further, consider operators $A, B, S, T: X \rightarrow X$ given by

$$
\left\{\begin{array}{l}
A h(x)=\sup _{y \in D}\left\{f(x, y)+G_{1}(x, y, h(\tau(x, y)))\right\} \\
B h(x)=\sup _{y \in D}\left\{f(x, y)+G_{2}(x, y, h(\tau(x, y)))\right\} \\
S h(x)=\sup _{y \in D}\left\{f(x, y)+G_{3}(x, y, h(\tau(x, y)))\right\} \\
T h(x)=\sup _{y \in D}\left\{f(x, y)+G_{4}(x, y, h(\tau(x, y)))\right\}
\end{array}\right.
$$

for $h \in X, x \in W$; these mappings are well-defined if the functions $f$ and $G_{i}$ are bounded.

Theorem 5.1. Let $A, B, S, T: X \rightarrow X$ be given by (5.2) and suppose that the following hypotheses hold:

(I) the functions $G_{i}: W \times D \times \mathbb{R} \rightarrow \mathbb{R}, i \in\{1,2,3,4\}$, satisfy

$$
\exp \left(-\frac{t}{\sup _{x \in W} \sup _{y \in D}\left|G_{1}(x, y, h(x))-G_{2}(x, y, k(x))\right|}\right)
$$


$\leq \phi\left(\max \left\{\begin{array}{c}\mathfrak{g}\left(F_{S h, T k}(t)\right), \mathfrak{g}\left(F_{S h, A h}(t)\right), \mathfrak{g}\left(F_{T k, B k}(t)\right), \\ \frac{1}{2}\left[\mathfrak{g}\left(F_{A h, S h}(t)\right)+\mathfrak{g}\left(F_{B k, T k}(t)\right)\right], \frac{1}{2}\left[\mathfrak{g}\left(F_{A h, S h}(t)\right)+\mathfrak{g}\left(F_{S h, T k}(t)\right)\right], \\ \frac{1}{2}\left[\mathfrak{g}\left(F_{B k, T k}(t)\right)+\mathfrak{g}\left(F_{S h, T k}(t)\right)\right], \frac{1}{2}\left[\mathfrak{g}\left(F_{S h, B k}(t)\right)+\mathfrak{g}\left(F_{T k, A h}(t)\right)\right]\end{array}\right\}\right)$, for all $h, k \in X$ and $t \in[0,1]$, where $\mathfrak{g}$ is given by $\mathfrak{g}(t)=1-t$ for $t \in[0,1]$;

(II) $f: W \times D \rightarrow \mathbb{R}$ and $G_{i}: W \times D \times \mathbb{R} \rightarrow \mathbb{R}$ are bounded functions, for $i \in\{1,2,3,4\}$;

(III) there exist sequences $\left\{h_{n}\right\}$ and $\left\{k_{n}\right\}$ in $X$ and $h^{*} \in X$ such that

$$
\lim _{n \rightarrow \infty} A h_{n}=\lim _{n \rightarrow \infty} B k_{n}=\lim _{n \rightarrow \infty} S h_{n}=\lim _{n \rightarrow \infty} T k_{n}=h^{*} ;
$$

(IV) $A S h=S A h$, whenever $A h=S h$ for some $h \in X$;

(V) $B T k=T B k$, whenever $B k=T k$ for some $k \in X$.

Then the system of functional equations (5.1) has a unique bounded solution.

Proof. Define

$$
F_{h, k}(t)= \begin{cases}1-\exp \left(-\frac{t}{d(h, k)}\right) & \text { if } 0<t \leq d(h, k), h \neq k, \\ 1 & \text { otherwise }\end{cases}
$$

where $h, k \in X$. Then $(X, \mathcal{F}, \mathcal{T})$ is a complete N. A. Menger PM-space (induced by the metric $d$ ) with $\mathcal{T}(a, b)=\min \{a, b\}$, for $a, b \in[0,1]$.

By hypothesis (III) the pairs $(A, S)$ and $(B, T)$ share the common limit range property with respect to $(S, T)$. Now, let $\epsilon$ be an arbitrary positive number, $x \in W$ and $h, k \in X$. Then there exist $y_{1}, y_{2} \in D$ such that

$$
\begin{aligned}
& A h(x)<f\left(x, y_{1}\right)+G_{1}\left(x, y_{1}, h\left(\tau\left(x, y_{1}\right)\right)\right)+\epsilon, \\
& A h(x) \geq f\left(x, y_{2}\right)+G_{1}\left(x, y_{2}, h\left(\tau\left(x, y_{2}\right)\right)\right), \\
& B k(x)<f\left(x, y_{2}\right)+G_{2}\left(x, y_{2}, k\left(\tau\left(x, y_{2}\right)\right)\right)+\epsilon, \\
& B k(x) \geq f\left(x, y_{1}\right)+G_{2}\left(x, y_{1}, k\left(\tau\left(x, y_{1}\right)\right)\right) .
\end{aligned}
$$

Using (5.3) and (5.6), we obtain

$$
\begin{aligned}
A h(x)-B k(x) & <G_{1}\left(x, y_{1}, h\left(\tau\left(x, y_{1}\right)\right)\right)-G_{2}\left(x, y_{1}, k\left(\tau\left(x, y_{1}\right)\right)\right)+\epsilon \\
& \leq\left|G_{1}\left(x, y_{1}, h\left(\tau\left(x, y_{1}\right)\right)\right)-G_{2}\left(x, y_{1}, k\left(\tau\left(x, y_{1}\right)\right)\right)\right|+\epsilon \\
& \leq \sup _{y \in D}\left|G_{1}(x, y, h(\tau(x, y)))-G_{2}(x, y, k(\tau(x, y)))\right|+\epsilon .
\end{aligned}
$$

Analogously, by using (5.4) and (5.5), we get

$$
B k(x)-A h(x)<\sup _{y \in D}\left|G_{1}(x, y, k(\tau(x, y)))-G_{2}(x, y, h(\tau(x, y)))\right|+\epsilon .
$$

From (5.7) and (5.8), we deduce that

$$
|A h(x)-B k(x)|<\sup _{y \in D}\left|G_{1}(x, y, h(\tau(x, y)))-G_{2}(x, y, k(\tau(x, y)))\right|+\epsilon,
$$

wherefrom it follows that

$$
d(A h, B k) \leq \sup _{x \in W} \sup _{y \in D}\left|G_{1}(x, y, h(\tau(x, y)))-G_{2}(x, y, k(\tau(x, y)))\right|+\epsilon .
$$


Since $\epsilon>0$ was taken arbitrary, we obtain that

$$
d(A h, B k) \leq \sup _{x \in W} \sup _{y \in D}\left|G_{1}(x, y, h(\tau(x, y)))-G_{2}(x, y, k(\tau(x, y)))\right| .
$$

In view of hypothesis (I) and (5.9), it follows easily that

$$
\begin{aligned}
& \mathfrak{g}\left(F_{A h, B k}(t)\right) \\
\leq & \phi\left(\max \left\{\begin{array}{c}
\mathfrak{g}\left(F_{S h, T k}(t)\right), \mathfrak{g}\left(F_{S h, A h}(t)\right), \mathfrak{g}\left(F_{T k, B k}(t)\right) \\
\frac{1}{2}\left[\mathfrak{g}\left(F_{A h, S h}(t)\right)+\mathfrak{g}\left(F_{B k, T k}(t)\right)\right], \frac{1}{2}\left[\mathfrak{g}\left(F_{A h, S H_{1}}(t)\right)+\mathfrak{g}\left(F_{S h, T k}(t)\right)\right], \\
\frac{1}{2}\left[\mathfrak{g}\left(F_{B k, T k}(t)\right)+\mathfrak{g}\left(F_{S h, T k}(t)\right)\right], \frac{1}{2}\left[\mathfrak{g}\left(F_{S h, B k}(t)\right)+\mathfrak{g}\left(F_{T k, A h}(t)\right)\right]
\end{array}\right\}\right) .
\end{aligned}
$$

Moreover, in view of hypotheses (IV) and (V), the pairs $(A, S)$ and $(B, T)$ are weakly compatible. Hence, Theorem 3.2 is applicable, and so $A, B, S$ and $T$ have a unique common fixed point, that is, the system of functional equations (5.1) has a unique bounded solution.

\section{Concluding remarks}

Coincidence and common fixed point results for a quadruple of self mappings satisfying common limit range property and weak compatibility under generalized $\Phi$-contractive conditions in Non-Archimedean Menger PM-spaces are proved. In particular, using common limit range property, conditions like continuity of mappings, closedness of the respective ranges, and containment of these ranges are completely avoided. A new result on the existence and uniqueness of solutions for certain system of functional equations arising in dynamic programming is obtained as a consequence.

Acknowledgement. The authors would like to thank Professor Sunny Chauhan and Professor Calogero Vetro for reprints of the papers [7, 8].

\section{References}

[1] M. Aamri and D. El. Moutawakil, Some new common fixed point theorems under strict contractive conditions, J. Math. Anal. Appl. 270 (2002), no. 1, 181-188.

[2] R. Bellman and E. S. Lee, Functional equations in dynamic programming, Aequationes Math. 17 (1978), no. 1, 1-18.

[3] T. C. Bhakta and S. Mitra, Some existence theorems for functional equations arising in dynamic programming, J. Math. Anal. Appl. 98 (1984), no. 2, 348-362.

[4] S. S. Chang, Fixed point theorems for single-valued and multi-valued mappings in NonArchimedean Menger probabilistic metric spaces, Math. Japon. 35 (1990), no. 5, 875885.

[5] S. S. Chang, Y. J. Chom, and S. M. Kang, Nonlinear Operator Theory in Probabilistic Metric Spaces, Nova Science Publishers, New York, 2001.

[6] S. Chauhan and S. Kumar, Fixed point theorems in Non-Archimedean Menger PMspaces using common property (E.A), Asian-Eur. J. Math. 5 (2012), no. 4, 13 pages.

[7] S. Chauhan, S. Radenović, M. Imdad, and C. Vetro, Some integral type fixed point theorems in Non-Archimedean Menger PM-Spaces with common property (E.A) and application to functional equations in dynamic programming, Rev. R. Acad. Cienc. Exactas Fís. Nat. Ser. A Math. 108 (2014), no. 2, 795-810. 
[8] S. Chauhan and J. Vujaković, Some fixed point theorems for weakly compatible mappings in Non-Archimedean Menger probabilistic metric spaces via common limit range property, Matematiche (Catania) 68 (2013), no. 2, 77-90.

[9] Y. J. Cho, S. M. Kang, and S. S. Chang, Coincidence point theorems for nonlinear hybrid contractions in Non-Archimedean Menger probabilistic metric spaces, Demonstratio Math. 28 (1995), no. 1, 19-32.

[10] - Common fixed point theorems for compatible mappings of type $(A)$ in NonArchimedean Menger PM-spaces, Math. Japon. 46 (1997), no. 1, 169-179.

[11] R. C. Dimri and B. D. Pant, Fixed point theorems in non-Archimedean Menger spaces, Kyungpook Math. J. 31 (1991), no. 1, 89-95.

[12] M. Grabiec, Y. J. Cho, and V. Radu, On Nonsymmetric Topological and Probabilistic Structures, Nova Science Publishers, New York, 2006.

[13] O. Hadžić, A note on Istrătescu's fixed point theorems in non-Archimedean probabilistic metric spaces, Bull. Math. Soc. Sci. Math. R. S. Roumanie (N.S.) 24(72) (1980), no. 4, 359-362.

[14] M. Imdad, J. Ali, and M. Tanveer, Coincidence and common fixed point theorems for nonlinear contractions in Menger PM spaces, Chaos Solitons Fractals 42 (2009), no. 5, 3121-3129.

[15] M. Imdad, S. Chauhan, and Z. Kadelburg, Fixed point theorems for mappings with common limit range property satisfying generalized $(\psi, \varphi)$-weak contractive conditions, Math. Sci. (Springer) 7 (2013), Art. 16, 8 pp.

[16] M. Imdad, S. Chauhan, Z. Kadelburg, and C. Vetro, Fixed point theorems for non-self mappings in symmetric spaces under $\varphi$-weak contractive conditions and an application to functional equations in dynamic programming, Appl. Math. Comput. 227 (2014), 469-479.

[17] M. Imdad, B. D. Pant, and S. Chauhan, Fixed point theorems in Menger spaces using the $\left(C L R_{S T}\right)$ property and applications, J. Nonlinear Anal. Optim. 3 (2012), no. 2, $225-237$.

[18] I. Istrătescu, On some fixed point theorems with applications to the nonarchimedean Menger spaces, Atti Accad. Naz. Lincei Rend. Cl. Sci. Fis. Mat. Natur. (8) 58 (1975), no. $3,374-379$.

[19] _ Fixed point theorems for some classes of contraction mappings on nonarchimedean probabilistic metric space, Publ. Math. Debrecen 25 (1978), no. 1-2, 29-34.

[20] I. Istrătescu and G. Babescu, On the completion on non-Archimedean probabilistic metric spaces, Seminar de spatii metrice probabiliste, Universitatea Timisoara, Nr. 17, 1979.

[21] I. Istrățescu and N. Crivat, On some classes of non-Archimedean probabilistic metric spaces, Seminar de spatii metrice probabiliste, Universitatea Timisoara, Nr. 12, 1974.

[22] I. Istrățescu and G. Palea, On non-Archimedean probabilistic metric spaces, An. Univ. Timişoara Ser. Şti. Mat. 12 (1974/77), no. 2, 115-118.

[23] G. Jungck, Common fixed points for noncontinuous nonself maps on nonmetric spaces, Far East J. Math. Sci. 4 (1996), no. 2, 199-215.

[24] G. Jungck and B. E. Rhoades, Fixed points for set valued functions without continuity, Indian J. Pure Appl. Math. 29 (1998), no. 3, 227-238.

[25] M. A. Khan, Common fixed point theorems in non-Archimedean Menger PM-spaces, Int. Math. Forum 6 (2011), no. 40, 1993-2000.

[26] M. A. Khan and Sumitra, A common fixed point theorem in non-Archimedean Menger PM-space, Novi Sad J. Math. 39 (2009), no. 1, 81-87.

[27] S. Kutukcu and S. Sharma, A common fixed point theorem in non-Archimedean Menger PM-spaces, Demonstratio Math. 42 (2009), no. 4, 837-849. 
[28] H. K. Pathak, Y. J. Cho, S. S. Chang, and S. M. Kang, Compatible mappings of type $(P)$ and fixed point theorems in metric spaces and probabilistic metric spaces, Novi Sad J. Math. 26 (1996), no. 2, 87-109.

[29] K. P. R. Rao and E. T. Ramudu, Common fixed point theorem for four mappings in non-Archimedean Menger PM-spaces, Filomat 20 (2006), no. 2, 107-113.

[30] B. Schweizer and A. Sklar, Statistical metric spaces, Pacific J. Math. 10 (1960), 313-334.

[31] V. M. Sehgal and A. T. Bharucha-Reid, Fixed points of contraction mappings on probabilistic metric spaces, Math. Systems Theory 6 (1972), 97-102.

[32] B. Singh, A. Jain, and P. Agarwal, Semi-compatibility in non-Archimedean Menger PM-space, Comment. Math. 49 (2009), no. 1, 15-25.

[33] B. Singh, A. Jain, and M. Jain, Compatible maps and fixed points in non-Archimedean Menger PM-spaces, Int. J. Contemp. Math. Sci. 6 (2011), no. 37-40, 1895-1905.

[34] S. L. Singh and B. D. Pant, Common fixed points of weakly commuting mappings on non-Archimedean Menger PM-spaces, Vikram J. Math. 6 (1987), 27-31.

[35] S. L. Singh, B. D. Pant, and S. Chauhan, Fixed point theorems in non-Archimedean Menger PM-spaces, J. Nonlinear Anal. Optim. 3 (2012), no. 2, 153-160.

[36] B. Singh, R. K. Sharma, and M. Sharma, Compatible maps of type $(P)$ and common fixed points in non-Archimedean Menger PM-spaces, Bull. Allahabad Math. Soc. 25 (2010), no. 1, 191-200.

[37] W. Sintunavarat and P. Kumam, Common fixed point theorems for a pair of weakly compatible mappings in fuzzy metric spaces, J. Appl. Math. 2011, Article ID 637958, 14 pages, 2011

[38] Common fixed points for R-weakly commuting in fuzzy metric spaces, Ann. Univ. Ferrara Sez. VII Sci. Mat. 58 (2012), no. 2, 389-406.

[39] C. Vetro, S. Chauhan, E. Karapınar, and W. Shatanawi, Fixed points of weakly compatible mappings satisfying generalized $\varphi$-weak contractions, Bull. Malaysian Math. Sci. Soc. (2014), in press.

Hemant Kumar Nashine

Department of Mathematics

Disha Institute of Management and Technology

SATYA Vihar, VidHANSABHA-ChANDRAKHURI MARG

MANDir HASAud, RAIPUR-492101(ChHATtisGarh), India

E-mail address: drhknashine@gmail.com, nashine_09@rediffmail.com

ZORAN KADELBURG

FACUlTy of Mathematics

UNIVERSITY OF BELGRADE

Studentski trg 16, 11000 Beograd, Serbia

E-mail address: kadelbur@matf.bg.ac.rs 